\title{
Reduction of peripheral blood stem cell collection sessions with extended-hour operation of the apheresis center
}

\begin{abstract}
Background: With the rising cost of healthcare, and nurses' salaries accounting for a large percentage of hospital expenses, it is crucial to maximize the efficiency of nursing services, while minimizing operational cost and improving quality of care.

Materials and methods: We implemented a scheduling modification with the potential to reduce the number of days patients undergo peripheral blood stem cell (PBSC) collection. The new schedule extended nurse's work-day hours, and nurses were staggered throughout the operational hours of the unit, allowing for extended collections. A retrospective analysis of 80 patients, mobilized by both G-CSF and plerixafor, and undergoing 158 autologous PBSC collection sessions, was performed to compare the operational data preceding and following this schedule change. The independent t-test and SPSS software were used for statistical analysis.
\end{abstract}

Results: Operational efficiency and quality improved after implementing the new schedule. More BVs were processed per day $(\mathrm{p}<0.001)$; collection days were reduced $(\mathrm{p}<0.001)$; an estimated $\$ 119,928$ (eight months) was saved; and patient and nurse satisfaction increased.

Discussion: Our study demonstrates that daily extended-hour PBSC collection facilitated by longer daily apheresis operation yielded the reduced number of PBSC collection sessions, a reduced cost, and improved quality of patient care.

Keywords: autografts, blood volume, cost savings, organization and administration, peripheral blood stem cell transplantation
Volume 3 Issue 2 - 2018

\author{
Ding Wen Wu,' SYoon Choo, ${ }^{2}$ Rachel \\ Friedmann, ${ }^{3}$ Adolfo Firpo-Betancourt, ${ }^{2}$ \\ Sharon Tindle, ${ }^{2}$ Godsfavour Guillet, ${ }^{4}$ \\ Tamarah Kent, ${ }^{4}$ Seby Jacob ${ }^{5}$ \\ 'Department of Pathology and Laboratory Medicine, NYU \\ Langone Health, NY, USA \\ ${ }^{2}$ Department of Pathology, Icahn School of Medicine at Mount \\ Sinai, NY, USA \\ ${ }^{3}$ Department of Pathology, Montefiore Medical Center, NY, USA \\ ${ }^{4}$ Apheresis Center, Mount Sinai Hospital, NY, USA \\ ${ }^{5}$ Department of Medicine, Nyack Hospital, NY, USA
}

Correspondence: Seby Jacob, MD, Department of Medicine, Nyack Hospital, Nyack, NY, USA, Email sebyjacob@gmail.com

Received: February 08, 2018 | Published: March 01, 2018
Abbreviations: $\mathrm{BV}$, blood volume; $\mathrm{PB} \mathrm{CD} 34+$, peripheral blood CD34+ cell count; MM, multiple myeloma; G-CSF, granulocyte colony stimulating factor; PY, product yield; PBSC, peripheral blood stem cell

\section{Introduction}

As healthcare expenses continue to increase, and healthcare funding becomes heavily reduced, hospitals are faced with the challenge of providing quality care while curtailing costs. ${ }^{1}$ Since nursing salaries account for the highest percentage of healthcare expenditure, many hospitals have restricted the size of their nursing workforce and restructured their rosters in a manner that would adequately allocate staff to meet patient needs, while simultaneously increasing operational efficiency. ${ }^{1,2}$ At the Mount Sinai Hospital Apheresis Center, full-time apheresis nurses previously worked on an 8.5-hour shift (8:00 am-4:30 pm), five days a week. Following the recommendation of a proposed guideline for autologous PBSC collection, the previous daily maximal processing volume for each patient was increased from 3 to 4 blood volumes (BV), except when the initial peripheral blood CD34+ cell count (PB CD34+) was $>120$ cells $/ \mathrm{mL} .^{3}$ The time required to process the $4 \mathrm{BVs}$ varies between patients, and between collection sessions, based upon flow rate, health condition of the patient, and mobilization efficiency. Four BVs was usually the maximal blood volume that could be processed within the 8.5-hour limit of daily operation of the Apheresis Center.

Autologous PBSC transplantation is performed to restore hematopoietic recovery in patients with hematological malignancies, including lymphomas, multiple myeloma (MM), Amyloidosis, and certain solid tumors, ${ }^{4}$ after high dose chemotherapy. PBSC collections are done in preparation for an autologous transplant, in which the stem cells are infused back into the patient. PBSC collection involves the collection of mobilized stem cells in the patient's blood that are used for hematopoietic reconstitution in stem cell transplantation. To maximize collection, patients undergo stem cell mobilizing regimens that consist of chemotherapy and/or granulocyte colony stimulating factor (G-CSF; filgrastim), with or without additional mobilizing agent, plerixafor, to mobilize the highest possible population of stem cells into blood circulation. ${ }^{4-6}$ the collection procedure is to process several blood volumes in an apheresis instrument, in order to reach an optimal target number of cells collected. ${ }^{7}$ The PBSC collection product yield (PY) rises with the increase of BVs processed. The number of collection days varies among patients, depending on the peripheral blood stem cell concentration and daily BV processed. 
The goal of collections is to collect an ideal target number of stem cells, which has been shown to achieve higher rates of prompt engraftment after transplantation. ${ }^{3,8,9}$ Peripheral CD34+ cells serve as a surrogate marker for hematopoietic stem cells, and its blood concentration is therefore used as a measurement to predict the PBSC collection yield of the same day. ${ }^{3,7}$ At the Mount Sinai Hospital, the minimum and optimal stem cell collection targets for autologous patients with a diagnosis of lymphoma are $2.5 \times 10^{6}$ and $5 \times 10^{6}$ CD34+ cells/kg body weight, respectively. For patients diagnosed with multiple myeloma, the optimal stem cell collection targets is $5 \mathrm{x}$ $10^{6}$ for a single transplant, and $10 \times 10^{6} \mathrm{CD} 34+$ cells $/ \mathrm{kg}$ for two tandem transplants. ${ }^{3,10-16}$ The apheresis service at our institution performs hundreds of PBSC collection procedures annually. Over the last few years, the overall demand for PBSC collection and other apheresis procedures amplified in our institution. Without a proportional increase in nursing staff, it is becoming ever more necessary to restructure the labor force, to more efficiently handle the growing caseload. We hypothesized that an extension of the daily operation hours might result in an improvement of service for patients who were mobilized for PBSC collection. In order to provide extended daily collections, the nurses' shifts were changed to 10.5 hours per day for 4 days a week, starting from August 2013. This change would potentially increase operational efficiency without requiring additional nursing staff. Under this new schedule, two nursing shifts were staggered on Monday-Friday, to cover an extended 12.5-hour day $(7: 30 \mathrm{am}$ to $8: 00 \mathrm{pm})$. Though the daily hours of operation in the unit increased, weekly working hours for each apheresis nurse remained unchanged. In order to assure the timely processing of the collected stem cells, the technologists' shifts of the Cellular Therapy Laboratory, which processes the stem cell collection products, were also staggered to extend operational hours. This allows the lab technologists to pick up the product of stem cell collection, which was completed in the evening, in a timely manner. However, there is only one transfusion medicine physician who covers the apheresis service on any given week. Therefore, the physician on service must work longer hours to cover the extended hours of the Apheresis Center for PBSC collections. Close cooperation among the apheresis nurses, transfusion medicine physicians, and the laboratory technologists is essential to extend the operational hours for PBSC collections. In this paper, we discuss the implementation of nursing schedule changes in the apheresis service of a large teaching hospital and its impact on PBSC collection operation. To our knowledge, the exploration of the operational benefits by changing the nurse scheduling in an apheresis service and the impact of the change on PBSC collection have not been reported.

\section{Materials and methods}

\section{Aim}

To investigate the clinical and economic benefits of changes in the nursing schedule on peripheral blood stem cell (PBSC) collection at the Mount Sinai Hospital.

\section{Study design}

A retrospective study design was employed to test the hypothesis that a schedule change would facilitate the processing of more BVs. For this purpose, we examined the mean number of $\mathrm{BV}$ processed for PBSC collection procedures performed before and after the schedule change. We also explored whether processing higher BVs would reduce the number of sessions needed to reach the clinically set target CD34+ counts. Exempt review of this study proposal was obtained from Institutional Review Board of Mount Sinai Medical Center.

\section{Organization of our apheresis service}

The apheresis team consists of: (1) Board certified transfusion medicine physicians, who are responsible for overseeing daily operations as well as managing all patients undergoing apheresis procedures; (2) A nurse manager and a nurse coordinator, who establish and update policies, processes and procedures, collect and review data from all procedures conducted, and ensure that safety and quality measures are met; and (3) Five full-time apheresis nurses, who coordinate patient scheduling, conduct pre-screening of patients before all scheduled procedures, perform apheresis procedures, and provide post-procedure instruction and care. All members of the inhouse apheresis team are salaried employees. Physicians refer patients for PBSC collection services by consulting transfusion medicine physicians, who evaluate the patient, determine the appropriateness of treatment, and tailor a PBSC collection plan. Prior to PBSC collection procedures, each patient undergoes a pre-screening session with one of the apheresis nurses. During this session, the nurse obtains a comprehensive medical history, evaluates the patient's peripheral veins for venous access, and when applicable coordinates the scheduling of central venous access placement with interventional radiology. PBSC collection procedures are mostly conducted as outpatient procedures in the Apheresis Center, a six bed unit that provides therapeutic apheresis services to children and adults. The requests for PBSC collection procedures were exclusively for adult autologous donors.

\section{Old nursing schedule}

Prior to August 2013, the apheresis service hours for PBSC collection were from 8:30 am to 4:30 pm, Monday-Friday, excluding holidays. This is based on the working schedule of full-time apheresis nurses at the Mount Sinai Hospital Apheresis Center (Figure 1). Only the nurses working in the Apheresis Center are qualified to perform PBSC collection at the Mount Sinai Hospital Apheresis Center per the Center's policy.

\section{New nursing schedule}

Under the new schedule, implemented starting on August 12, 2013, the full-time nurse's hours were extended to 10.5 hours per day, including a 30-minute lunch break, for four days a week, with one day off during the week. The 40-hour work week was not changed. Unlike the previous schedule, however, two daily shifts were introduced, with nurses staggered. With this new setup, nurses who began working on a later shift could take over the care of patients who started collection with the early shift nurses (Figure 2).

\section{Patient population and PBSC mobilization}

We conducted a retrospective analysis of the PBSC collection cases, performed from January 2, 2013 to April 30, 2014. A total of 529 PBSC collection procedures were performed during the specified time. Procedures performed prior to the schedule change were assigned to the pre-change group, and those performed after the schedule change was assigned to the post-change group. The cases completed in the month of August were excluded from our analysis, so that the transition period between schedules would not 
affect our assessment. All patients received 5 daily doses $(8-10 \mu \mathrm{g} / \mathrm{kg})$ of G-CSF and one dose of plerixafor $(0.24 \mathrm{mg} / \mathrm{kg})$ the night before starting stem cell collection. Both medications were continued prior to each planned apheresis session. A patient's first leukapheresis session was scheduled on the 5th day of G-CSF administration, which usually correlates with peak PBSC concentration. ${ }^{3,17,18}$ Plerixafor was administered on the night of G-CSF day four and continued before each leukapheresis session. Citrate, used for anticoagulation, could cause higher risks of toxicity and hypocalcemia, with increasing BV processing. To ensure patient comfort and safety, an increased dose of intravenous calcium gluconate was given prophylactically to prevent hypocalcemia in higher BV processing. ${ }^{19}$

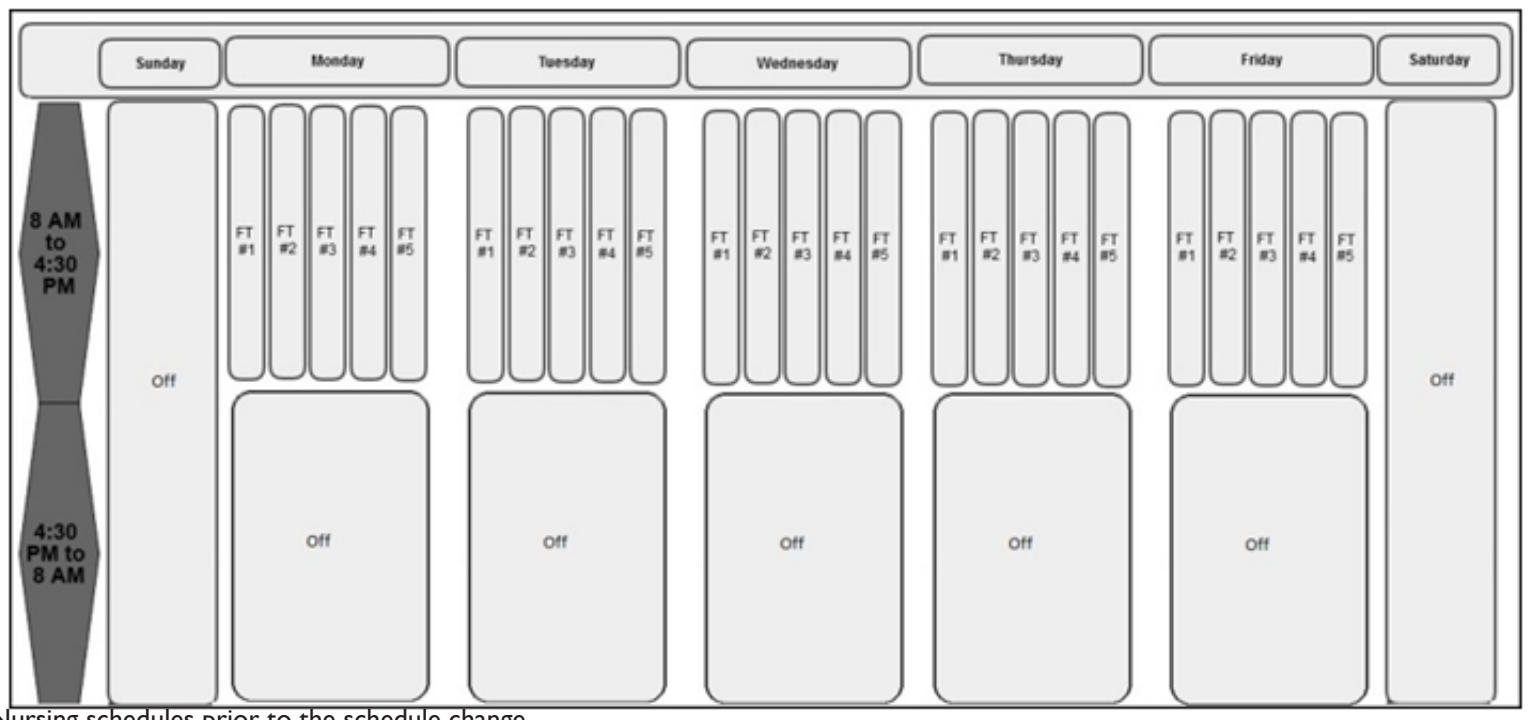

Figure I Nursing schedules prior to the schedule change.

At our Apheresis Center, full-time apheresis nurses previously worked on an 8.5-hour shift (8:00 am - 4:30 pm), five days a week. Accordingly, the operation hours of our Apheresis Center was from 8:00am - 4:30 pm on weekdays.

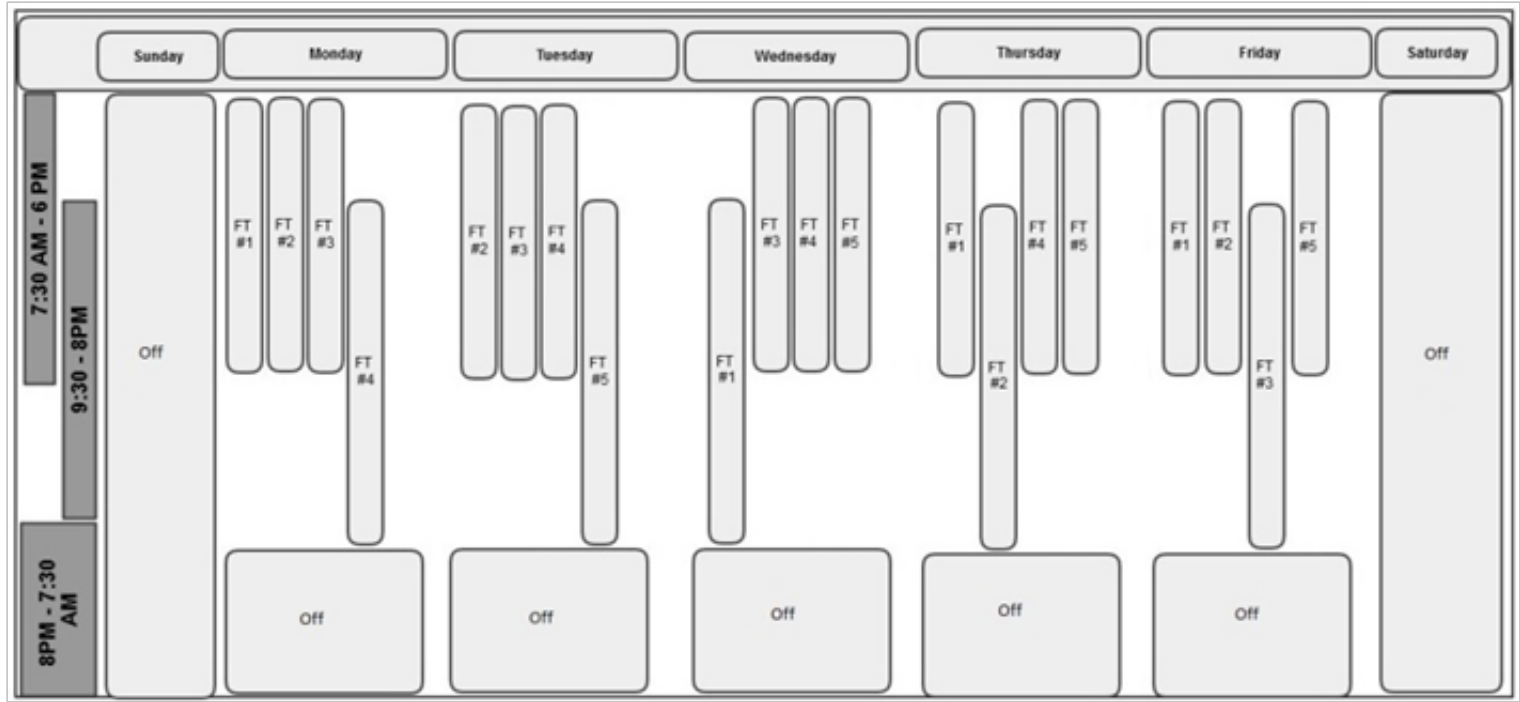

Figure 2 Nursing schedule after restructuring.

Under the new schedule, the full-time nurse's hours were extended to 10.5 hours per day for four days a week, with one day off during the week. The 40hour work week was not changed. Unlike the previous schedule, however, two daily shifts were introduced, with nurses staggered. The operation hours were extended from previous 8.5 hours to current 12.5 hours.

\section{Inclusion criteria}

The following collections were included in the study:
I. Autologous PBSC collection.
II. Adult patients.

III. PBSC collection during the period between Jan. 2, 2013 and Apr. 30, 2014.

IV. Patients were mobilized by both G-CSF and plerixafor.

V. PB CD34+ cell count on the day of collection was between 10$120 / \mu \mathrm{L}$. 


\section{Exclusion criteria}

Patients with collections from July 30, 2013 to August 31, 2013, the transition month of the nursing schedule change. This transition period thus would not affect our assessment.

A. Patients with PB CD34 $+<10 / \mu \mathrm{L}$ or $>120 / \mu \mathrm{L}$ (because extension of operation hours has little impact for collections of these patients).

B. Patients mobilized in conjunction with chemotherapy.

C. Patients who arrived late on the collection day due to various causes, such as insertion of a central venous catheter in the morning, etc.

D. PBSC collection was not completed and the collection target was not reached due to various reasons.

\section{Stem cell collection}

The PBSC collections were performed for both patients with scheduled or even non-scheduled (potential) hematopoietic stem cell transplants. At our institution, the minimum target and optimal target of each stem cell collection for autologous patients with diagnosis of lymphomas are $2.5 \times 10^{6}$ and $5 \times 10^{6} \mathrm{CD} 34+$ cells $/ \mathrm{kg}$ body weight, respectively. The optimal target for patients with multiple myeloma $(\mathrm{MM})$ is $5 \times 10^{6}$ for a single transplant, and $10 \times 10^{6} \mathrm{CD} 34+$ cells/ $\mathrm{kg}$ for a tandem transplant. ${ }^{12,20}$ Peripheral blood mononucleated cell leukapheresis was performed for PBSC collection using Terumo BCT COBE Spectra Apheresis System (Lakewood, CO, USA). The PBSC collections were performed via peripheral venous access or centrally placed double-lumen catheters. Extracorporeal anticoagulation was achieved with anticoagulant citrate dextrose-A (ACD-A), and citrate-induced hypocalcemia was prevented and/or managed with intravenous calcium gluconate supplementation. ${ }^{19}$ Volume of blood processed for PBSC collection and volume of collection were computed by the COBE Spectra apparatus.

\section{Measurement of CD34+ cell counts}

Flow cytometry measurement of CD34+ stem cell counts were performed by the in-house Flow Cytometry Laboratory using the Stem-Kit ${ }^{\mathrm{TM}} \mathrm{CD} 34+$ HPC Enumeration Kit (Beckman Coulter, FL, USA), applying a single-platform modified ISHAGE protocol. Automated stem cell counts were performed on the Beckman Coulter Gallios Cytometer (Beckman Coulter, FL, USA). Flow cytometry was performed on a $10 \mathrm{~mL}$ sample of pre-apheresis heparinized peripheral blood to determine the PB CD34+ cell count. A $0.5-1 \mathrm{~mL}$ EDTA anticoagulated sample of the apheresis collection product was drawn after each collection session to determine the collection product CD34+ yield (PY). The PY was sampled at our Cellular Therapy Laboratory. The pre-collection sample for PB CD34+ was taken by the apheresis nurses. Each sample was submitted to the Flow Cytometry Laboratory. Estimation of daily PY (CD34+ cells x10 $/ \mathrm{kg})$ with $3 \mathrm{BV}$ processing was made by dividing the PB CD34+ count by a factor of 10 , multiplying by $10^{6}$, and dividing by the patient's weight in kg per Chepovetsky et al., ${ }^{3}$ for example, for a PB CD34+ count of 40 cells $/ \mu \mathrm{L}$ on a given day, the average collection yield is estimated to be around $4 \times 10^{6} \mathrm{CD} 34+$ cells $/ \mathrm{kg}$, if $3 \mathrm{BVs}$ are processed. To increase $\mathrm{PY}$ to $>8 \times 10^{6} \mathrm{CD} 34+$ cells $/ \mathrm{kg}, 6 \mathrm{BV}$ need to be processed on that day.

\section{End points of this study}

Daily blood volume processed per collection, number of blood volumes (BV) per day, reduction of collection days, number (and \%) of patients who benefited from the reduction of collection days. The blood volume processed per collection was measured by machine.

Blood volume processed $=($ Final inlet flow volume $)-($ final anticoagulation volume) displayed at the end of the collection session by the COBE Spectra Apheresis System.

$\#$ of CD34 + cells collected $=(C D 34+$ cell concentration of the product) $\mathrm{x}$ (product volume)

\section{Calculation for number of BV}

The calculation of BV is based on the manufacturer's manual:

$\#$ of $\mathrm{BV}=($ Blood volume processed $) \div$ (Patient's blood volume $)$.

\section{Calculation for collection days reduced}

The stem cell collection target was based on the diagnosis of each patient. PB CD34+ cell concentration was used to estimate daily collection PY (CD34+ cells/ $\mathrm{kg}$ ) for each PBSC collection day, if 3 - 4 BV per day is processed. ${ }^{3}$ In recent years, our effort to increase the daily collection yield was achieved by the maximal number of BV approaching 4. We thus calculate the reduction of collection days based on $4 \mathrm{BV}$ a day * as follows:

Predicted \# of collection days $*$ = total $\#$ of $\mathrm{BV}$ required (in the entire collection cycle, to reach the optimal target $) \div(4 \mathrm{BV} /$ day $)$

Reduction of collection days $=$ predicted \# of collection days*_ actual \# of collection days

\section{Determination of number of patients with collection days reduced}

Based on the calculated reduction of collection days mentioned above, the number of patients who had reduced collection days was counted manually.

\section{Statistics}

SPSS software was used for statistical analyses to compare the numbers of BV and the collection session reduction, before and after the nursing schedule change. The independent t-test was used for the majority of statistical analyses.

\section{Results}

A total of 529 PBSC collection procedures (i.e. sessions) were performed during the study period, however, 158 autologous PBSC collection procedures performed for 80 patients, met the inclusion criteria, and were thus included in our analysis. On the old schedule, 37 patients underwent 74 stem cell collection sessions, and on the new schedule, 43 patients had 84 sessions. The average BV processed under the old schedule was 14.96 liters compared to 17.41 liters processed from patients under the new schedule. This increase in processed BV was significant $(\mathrm{p}<0.0001)$. With increased BVs processed per session, collection days were reduced for $28 \%$ of patients. Total savings realized in the operation of the Apheresis Center were calculated at $\$ 119,928$ resulting from reductions in costs 
of mobilization, collection, and processing of PBSC. Physicians' fees were not included in calculations.

\section{Discussion}

We conducted a retrospective study to analyze the beneficial effects of modifying the nursing schedule to allow for extended PBSC collection operations. We found that this change had a multitude of effects benefiting patient care and costs.

\section{Benefits of collection day reduction}

Combination of G-CSF and plerixafor is known to be the most effective mobilization regimen for autologous PBSC collection. With this mobilization strategy, many patients only require 1-2 days of stem cell collection. However, there are drawbacks to this mobilization regimen, mainly uncomfortable side effects, such as bone pain (82\%), nausea $(10 \%)$, and diarrhea $(33 \%))^{4,21}$ An extended daily collection period, where an increased number of BVs are processed, would limit the number of collection days, thus limiting the administration of the mobilizing agents. The decreased number of collection days presents several additional benefits:

A. In addition to the discomfort caused by the mobilizing agents, the central venous catheter is often inserted into the internal jugular vein in the neck, which is not comfortable. ${ }^{3}$ With decreased number of collection days, the patient could have the catheter removed sooner.

B. As most patients have PBSC collection procedures done as outpatients, they and their caretakers need to travel to the hospital daily. The patients are usually advised not to travel via public transportation for PBSC collection, in order to avoid infection through the venous catheter. If patients live far away from the hospital, they often stay in a hotel nearby. Fewer collection days would decrease the amount of travel time, patient's expenses, and hotel stay (if needed). ${ }^{3}$

C. With longer daily collections, more BVs could be processed, which would help reach the collection target sooner. With the reduction of collection days, a greater number of patients could be scheduled for PBSC collection each week. Patients could begin collection as late as Wednesday or Thursday, and still meet the target for collection.

\section{Hospital financial savings}

As healthcare costs continue to rise at a faster pace than healthcare funding, medical facilities are faced with the challenge of providing excellent care with limited resources. Determining optimal nursing schedules has been one of the prime challenges in cost cutting. Here we explored the impact of restructuring nursing schedules in our apheresis service to meet these goals. The reduction of days needed to reach target collection counts in a subset of PBSC collection patients has very important implications. Minimizing PBSC collection days would bring down costs for the hospital, as there are specific expenses (e.g., labor, apheresis kits, reagents, testing and processing) associated with each procedure. Based on prior cost-analysis literature, we calculated that on average, the costs of mobilization, collection, and processing of peripheral blood stem cells for PBSC collection are $\$ 6,250, \$ 2,990$, and $\$ 754$, respectively. These costs accumulate with the increase in the number of mobilizations and collections; therefore, methods to achieve target $\mathrm{CD} 34+$ cell collections in as few collections as possible would result in significant cost reduction. ${ }^{22}$ It is important to recognize that our calculation did not include physicians' professional fees, since physicians are salaried workers at our hospital, and do not charge each patient. Without these items included, we calculated a savings of $\$ 119,928$ within eight months alone.

\section{Study size and average BV processed}

After the operation hours of the Apheresis Center were extended, transfusion medicine physicians worked with apheresis nurses to maximally increase daily blood processing volume up to $6 \mathrm{BV}$, when feasible. However, we were not able to process the maximal $6 \mathrm{BV}$ in a larger proportion of procedures, because of the following reasons:

Processing maximal BV is not feasible every day. The collection sessions of each collection cycle are often shortened by catheter insertion and removal procedures, patient's travel plans, transportation difficulties, or inclement weather, etc. Thus, the number of BV could not be increased to the maximal level on these days. Increasing the maximal number of BVs processed to reduce PBSC collection days was not an administrative decision from the leadership, but only at the individual physician's discretion, and required the voluntary efforts of the apheresis physicians and nurses. Therefore, there were no coordinated efforts to increase daily PBSC collection after the newly changed nursing schedule. Since this change was new for clinical staff and in some cases unfamiliar, we observed a reluctance to increase BV per session. With a consistent effort and coordination, the maximal number of daily $\mathrm{BV}$ could be achieved in a larger proportion of patients or sessions. Nevertheless, our study results showed that the new nursing schedule significantly increased number of BV per collection day $(\mathrm{p}<0.0001)$, thereby increasing the maximal daily collection yield, and reducing the number of collection days ( $<<0.001$ ). We observed an increase in the number of autologous PBSC patients who were able to benefit from reduction of collection days $(p<0.001)$. We were able to decrease discomfort, inconvenience, personal expenses, and travel time associated with the stem cell collection and mobilization for the patients. Through implementation of this modified schedule, we saved money for both our patients and the institution. We improved patient satisfaction, while maintaining the same number of weekly working hours of each nurse to ensure the safety and quality of apheresis nursing care.

\section{A 10.5-hour shift}

Given the positive results and cost saving seen with extending hours, we postulated that a schedule of full-time nurses with extended hours, possibly even up to 12-hour shifts may be an even more successful arrangement. However, many studies have suggested that 12-hour nursing shifts are associated with burnout and possible safety risk for patients. ${ }^{23,24}$ Therefore, our nursing shifts were not extended beyond 10.5 hours, with the goal of maintaining the greatest quality of care for our patients.

\section{Conclusion}

Through effective management and nursing schedule modification, we were able to provide patients with the opportunity to undergo reduced number of PBSC collection days by daily processing of increased $\mathrm{BVs}$, while minimizing discomfort, improving quality of care, and increasing financial savings. To our knowledge, this is 
the first study which examines the operational impact of a nursing schedule change on PBSC collection efficiency. ${ }^{25}$ This study is unique in that all the patients were mobilized by both G-CSF and plerixafor.
A larger study in which more patients have 4-6 BVs processed per session would likely show a greater reduction in the number of collection days (Table 1).

Table I Impact of Extended - Hour Operation on PBSC Collection: Post-change results compared to baseline pre-change data

\begin{tabular}{|c|c|c|c|}
\hline & $\begin{array}{l}\text { Pre-change ( } 74 \text { sessions/ } 37 \\
\text { patients) }\end{array}$ & $\begin{array}{l}\text { Post-change }(84 \text { sessions/ } 43 \\
\text { patients) }\end{array}$ & $p$ value \\
\hline Average daily blood volume processed (mL) & $14,956 \pm 3,893$ & $17,405 \pm 4,105$ & $<0.0001$ \\
\hline Average daily BV & $3.2 \pm 0.7$ & $3.9 \pm 0.8$ & $<0.0001$ \\
\hline \# of collection days reduced (with $4.0 \mathrm{BV} /$ day for comparison) & 0 & 12 & $<0.001$ \\
\hline $\begin{array}{l}\% \text { of collection sessions reduced from the predicted collection } \\
\text { sessions with } 4.0 \mathrm{BV} / \text { day }\end{array}$ & $0 \%$ & $14 \%$ & $\begin{array}{l}\text { Not } \\
\text { Applicable }\end{array}$ \\
\hline \# of patients having collection days reduced & 0 & 12 & $<0.001$ \\
\hline$\%$ of patients having collection days reduced & $0 \%$ & $28 \%$ & $\begin{array}{l}\text { Not } \\
\text { Applicable }\end{array}$ \\
\hline Cost saving ( 8 months) & - & $\$ 119,928$ & - \\
\hline
\end{tabular}

\section{Acknowledgements}

The authors are indebted to the nursing leadership for the strategic change of the apheresis nurse schedule in attempt of performance improvement. We acknowledge the apheresis nursing staff members for their carrying out all the stem cell collection procedures in the study. We are grateful to Dr. Loraine Miller and her Flow Cytometry Laboratory staff members for easy access and fast turnaround time of CD34+ count tests in their Laboratory. We thank Ms. Yelena Sinitsyn and her Cellular Therapy Laboratory staff members for their extending operation hours to collaborate with the nursing schedule change.

\section{Conflict of interest}

The authors have no conflicts of interest to declare.

\section{References}

1. Thungjaroenkul P, Cummings GG, Embleton A. The impact of nurse staffing on hospital costs and patient length of stay: a systematic review. Nurs Econ. 2007;25(5):255-265.

2. Penner SJ. Economics and financial management for nurses and nurse leaders. 2nd ed. New York: Springer Publishing Company; 2013.

3. Chepovetsky J, Yoon SC, Blouin AG. et al. Roles of peripheral blood CD34+ cell count and midpoint collection CD34+ cell yield for peripheral blood stem cell collections from autologous patients mobilized by $\mathrm{G}-$ CSF and plerixafor. $N A J$ Med Sci. 2013;6(2):63-70.

4. Kessans MR, Gatesman ML, Kockler DR. Plerixafor: a peripheral blood stem cell mobilizer. Pharmacotherapy. 2010;30(5):485-492.

5. Andreola G, Babic A, Rabascio C, et al. Plerixafor and Filgrastim XM02 (TevaGrastim) as a first line peripheral blood stem cel mobilisation strategy in patients with multiple myeloma and lymphoma candidates to autologous bone marrow transplantation. Eur J Haematol. 2012;88(2):154-158.

6. Flomenberg N, Devine SM, Dipersio JF, et al. The use of AMD3100 plus G-CSF for autologous hematopoietic progenitor cell mobilization is superior to G-CSF alone. Blood. 2005;106(5):1867-1874.

7. Bender JG, To LB, Williams S, et al. Defining a therapeutic dose of peripheral blood stem cells. J Hematother. 1992;1(4):329-341.
8. Berenson RJ, Bensinger WI, Hill RS, et al. Engraftment after infusion of CD34+ marrow cells in patients with breast cancer or neuroblastoma. Blood. 1991;77:1717.

9. Bensinger WI, Berenson RJ, Andrews RG. Engraftment after infusion of CD34 enriched marrow cells. Int $J$ of Cell Clon. 1992;10(Suppl S1):176-178.

10. Demirer T, Buckner CD, Gooley T, et al. Factors influencing collection of peripheral blood stem cells in patients with multiple myeloma. Bone Marrow Transplant. 1996;7(6):937-41.

11. Giralt S, Stadtmauer EA, Harousseau JL, et al. International myeloma working group (IMWG) consensus statement and guidelines regarding the current status of stem cell collection and high-dose therapy for multiple myeloma and the role of plerixafor (AMD 3100). Leukemia. 2009;23(10):1904-12.

12. Tricot G, Jagannath S, Vesole D, et al. Peripheral blood stem cell transplants for multiple myeloma: identification of favorable variables for rapid engraftment in 225 patients. Blood. 1995;85(2):588-596.

13. Pusic I, Jiang SY, Landua S, et al. Impact of mobilization and remobilization strategies on achieving sufficient stem cell yields for autologous transplantation. Biol Blood Marrow Transplant. 2008;14:1045-1056.

14. Sugrue MW, Williams K, Pollack BH, et al. Characterization and outcome of "hard to mobilize" lymphoma patients undergoing autologous stem cell transplantation. Leuk Lymphoma. 2000;39:509-519.

15. Bensinger W, Appelbaum F, Rowley S, et al. Factors that influence collection and engraftment of autologous peripheral-blood stem cells. $J$ Clin Oncol. 1995;13:2547-2555.

16. Mavroudis D, Read E, Cottler-Fox M. CD34+ cell dose predicts survival, post-transplant morbidity, and rate of hematologic recovery after allogeneic marrow transplants for hematologic malignancies. Blood. 1996;88:3223-3229.

17. Sinha S, Gastineau D, Micallef I, et al. Predicting PBSC harvest failure using circulating CD34 levels: developing target-based cutoff points for early intervention. Bone Marrow Transplant. 2011;46(7):943-949.

18. To LB, Levesque JP, Herbert KE. How I treat patients who mobilize hematopoietic stem cells poorly. Blood. 2011;118:4530-4540. 
19. Hedge V, Setia R, Soni S, et al. Prophylactic low dose continuous calcium infusion during peripheral blood stem cell (PBSC) collections to reduce citrate related toxicity. Transfus Apher Science. 2016;54(3):373-6.

20. Reik RA, Noto TA, Fernandez HF. Safety of large-volume leukapheresis for collection of peripheral blood progenitor cells. J Clin Apher. 1997;12(1):10-13.

21. Anderlini P, Przepiorka D, Seong D, et al. Clinical toxicity and laboratory effects of granulocyte-colony-stimulating factor (filgrastim) mobilization and blood stem cell apheresis from normal donors, and analysis of charges for the procedures. Transfusion. 1996;36(7):590-595.

22. Shaughnessy P, Islas-Ohlmayer M, Murphy J, et al. Cost and clinical analysis of autologous hematopoietic stem cell mobilization with G-CSF and plerixafor compared to G-CSF and cyclophosphamide. Biology of Blood and Marrow Transplantation. 2011;17(5):729-736.
23. Dall Ora C, Griffiths P, Ball J, et al. Association of $12 \mathrm{~h}$ shifts and nurses' job satisfaction, burnout and intention to leave: findings from a crosssectional study of 12 European countries. BMJ Open. 2015;5(9):e008331.

24. Griffiths P, Dall Ora C, Simon M, et al. Nurses' shift length and overtime working in 12 European countries: the association with perceived quality of care and patient safety. Med Care. 2014;52(11):975-81.

25. Jacob S, Choo SY, Friedmann R, et al. Impact of Nursing Schedule Change on Therapeutic Apheresis Service. Nursing Economic. 2017;35(4):218-222. 